\title{
Network-Aware Peer-to-Peer (P2P) and Internet Video
}

\author{
John F. Buford, ${ }^{1}$ Ken Kerpez, ${ }^{2}$ Yuanqiu Luo, ${ }^{3}$ Dave Marples, ${ }^{4}$ and Stan Moyer ${ }^{2}$ \\ ${ }^{1}$ Avaya Labs Research, 233 Mount Airy Road, Room 2A11, Basking Ridge, NJ 07920-2311, USA \\ ${ }^{2}$ Telcordia Technologies, 1 Telcordia Drive, Piscataway, NJ 08854, USA \\ ${ }^{3}$ Huawei Technologies USA, 400 Somerset Corporate Boulevard, Suite 602, Bridgewater, NJ 08807, USA \\ ${ }^{4}$ Technolution B.V., P. O. Box 9202, Mansfield Notts NG18 9DY, UK
}

Correspondence should be addressed to Ken Kerpez, kkerpez@telcordia.com

Received 10 March 2010; Accepted 10 March 2010

Copyright (c) 2010 John F. Buford et al. This is an open access article distributed under the Creative Commons Attribution License, which permits unrestricted use, distribution, and reproduction in any medium, provided the original work is properly cited.

Video and peer-to-peer content are both rapidly increasing Internet bandwidth demands. The recent reports predict an "exaflood" from advances in video over the Internet, rich media content, and user-generated content (UGC). Another trend is the continued popularity and growth of peer-topeer ( $\mathrm{P} 2 \mathrm{P})$ content delivery. Many estimates say that P2P accounts for most of the current Internet traffic, and that video accounts for most of the growth in Internet traffic. New systems and studies to optimize future P2P and video traffic can have a very high impact on the future of the Internet.

In many cases, $\mathrm{P} 2 \mathrm{P}$ traffic traverses long distances, across core networks and multiple Internet Service Provider (ISP) networks, even though the content could have been retrieved from a much closer location. Internet video is similar; often delivered from distant servers via multiple, redundant, unicast streams. To address this, there has been a spate of recent effort on systems, information exchange, and control to enable efficient P2P and video content distribution. New systems and protocols are needed to enable Internet content to work in concert with the network to be delivered from the best source or over the least congested links. Localized P2P traffic may only traverse a few hops instead of ten or twenty, allowing a vast decrease in core network bandwidth. Real-time streaming via $\mathrm{P} 2 \mathrm{P}$ is becoming competitive with traditional broadcast, and enhanced live P2P protocols are emerging. Related fields such as automotive telematics are embracing P2P techniques. Efficient measurement and analyses are necessary to understand new P2P protocols.

These topics and more are investigated in this special issue. Many thanks go to the paper authors and guest editors for providing the timely and high-quality analyses here. A summary of papers is as follows.
"RTSP-based mobile peer-to-peer streaming system," by Jani Antero Peltotalo et al., presents an effective real-time peer-to-peer streaming system for the mobile environment. The basis for the system is a scalable overlay network which groups peers into clusters according to their proximity using round-trip time (RTT) values between peers. Media is delivered using a number of "partial streams."

"Localized multistreams for P2P streaming," by Majed Alhaisoni et al., looks at the combination of two P2P streaming techniques, redundant streaming, and locality awareness, in the context of both live and video-on-demand streaming. Results show that redundancy affects network utilization only marginally if traffic is kept at the edges via localization techniques.

"Bandwidth reduction via localized peer-to-peer (P2P) video," by Ken Kerpez et al., presents recent research into P2P distribution of video that can be highly localized, preferably sharing content among users on the same access network and Central Office (CO). Results show that nearly all of the traffic volume of unicast video could be delivered via localized P2P.

"'Q-Feed"-an effective solution for the free-riding problem in unstructured $\mathrm{P} 2 \mathrm{P}$ networks," by $\mathrm{S}$. M. Thampi and Chandra Sekaran K, presents a solution for reducing the ill effects of free riders in decentralised unstructured P2P networks. "Q-learning" is applied to a free-riding control mechanism, which is shown to effectively service what is received by free riders and also encourage the lowperforming neighbors to improve performance with a higher quantity of popular files.

"A hybrid approach to assess the network awareness of P2P-TV applications," by D. Rossi and P. Veglia, develops a general methodology to assess the level of network awareness 
and friendliness of P2P-TV applications that is based on a combination of active and passive measurement techniques. This methodology is applied to PPLive, where it is shown that PPLive generally does not show preference toward peer proximity.

"Providing adapted contextual information in an overlay vehicular network," by Jose Santa et al., discusses P2P in the context of automotive telematics. The vehicular network presented in this paper fills the current gap between solutions lacking in flexibility, mainly supported by an infrastructure deployment, and those highly local and distributed, such as sole- vehicular ad hoc network (VANET) approximations.

\footnotetext{
John F. Buford Ken Kerpez Yuanqiu Luo Dave Marples Stan Moyer
} 

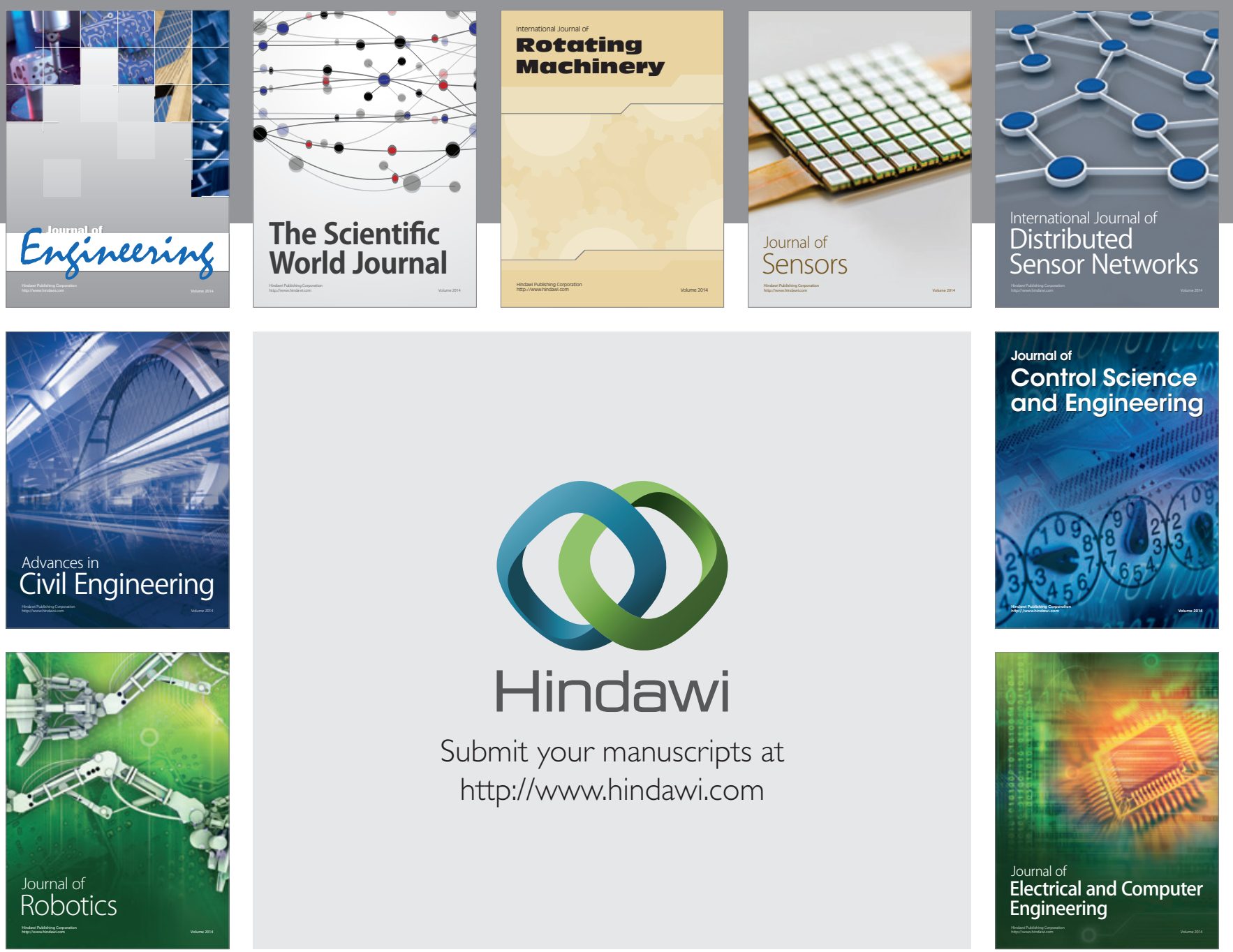

Submit your manuscripts at

http://www.hindawi.com
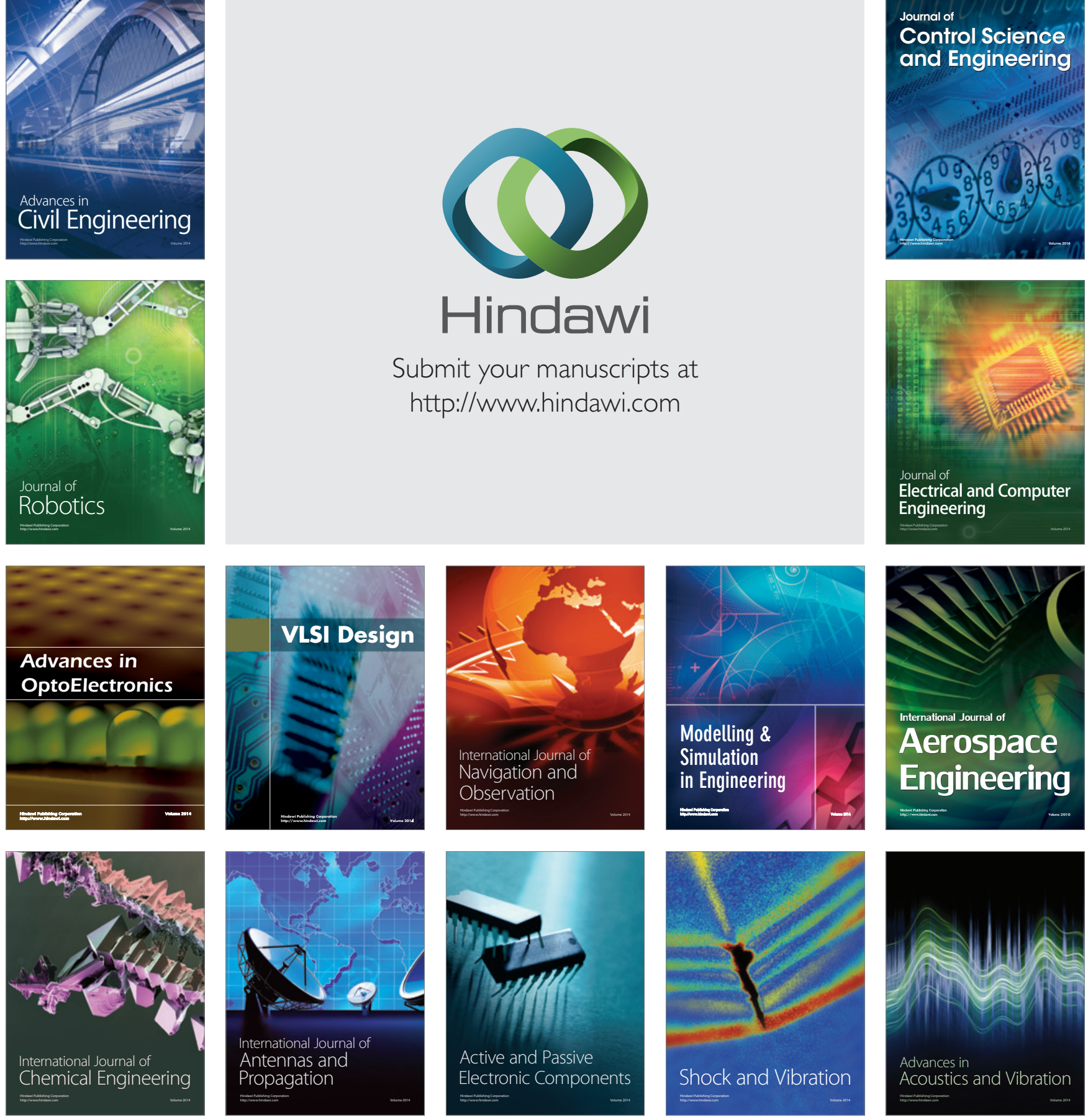